\title{
Einfluss der Naturschutzgesetzgebung auf die Forstgesetzgebung in Flandern
}

\author{
LUST, N., NACHTERGALE, L. \& SERBRUYNS, I.
}

Laboratorium für Forstwirtschaft, Universität von Gent, Geraardsbergsesteenweg 267, 9090 Melle, Belgium

Corresponding author

Tel ++32/9/2522113; fax ++32/9/2525466, e-mail: noël.lust@rug.ac.be

\section{Zusammenfassung}

Gegenstand dieser Untersuchung ist der Einfluss der Naturschutzgesetzgebung auf die Forstgesetzgebung in Flandern. Von großer Bedeutung hierfür ist zunächst das erste Naturschutzgesetz von 1973. Die Forstverwaltung hat dieses grob vernachlässigt und sah sich mit den entsprechenden Konsequenzen konfrontiert. 1990 wurde das erste Flämische Forstgesetz erlassen, in dem der ökologische Wert der Wälder bereits stark betont wurde. Das Flämische Naturschutzgesetz von 1997 hat jedoch den Einfluss des Naturschutzes auf den Wald noch wesentlich verstärkt. Und schliesslich haben die Änderungen des Flämischen Waldgesetzes von 1999 die Ökologie noch stärker gewichtet.

Die allgemeinen Schlussfolgerungen bezüglich des Verhältnisses zwischen Naturschutzgesetz und Waldgesetz sind offensichtlich. Es gibt eine Reihe von Überlappungen zwischen dem Waldgesetz von 1990, revidiert im Jahre 1999, und dem Naturschutzgesetz von 1997. Durch das Waldgesetz wird der Naturschutzwert des Waldes sehr stark betont. Der Einfluss des Naturschutzes auf die Forstpolitik, die Forstgesetzgebung und die Waldbewirtschaftung ist groß. Dabei kann das Naturschutzgesetz in Zukunft einen noch viel grösseren Einfluss auf die Waldbewirtschaftung ausüben. Durch die Naturschutzgesetzgebung gibt es zahlreiche Konfliktquellen zwischen dem Waldsektor und dem Naturschutzsektor. Sie können zu einer scharfen Auseinandersetzung zwischen beiden Gruppen führen, von der vor allem die Privatwaldbesitzer betroffen wären. Konflikte zwischen den Sektoren werden unvermeidlich zu einer Demotivation der Waldeigentümer und damit auch zu einem verminderten Wert des Waldbesitzes führen.

Schüsselwörter: Forstgesetzgebung, Naturschutzgesetzgebung.

\section{Einleitung}

Es ist üblich und erwünscht dass gesetzliche Aspekte hinsichtlich des Waldes in einer Forstgesetzgebung zusammengebracht werden. Auf diese Weise entsteht ein gebrauchsfreundliches und kohärentes Dokument. Es gibt jedoch noch eine Vielzahl von Regelungen die eingehend in die gesetzlichen Regelungen zum Wald eingreifen, dennoch aber in anderen Gesetzgebungen aufgenommen wurden. Dies führt zu allerlei Schwierigkeiten: 
- Es ist nicht hinreichend bekannt welche Regelungen in den verschiedenen Gesetzgebungen aufgenommen wurden.

- Die verschiedenen Regelungen sind nicht kohärent: einige widersprechen sich gegenseitig, andere sind zumindest verwirrend, wiederum andere Regelungen können auch als komplementär betrachtet werden.

- Allerlei Konfikte zwischen den verschiedenen Verwaltungen entstehen.

Dies alles hat zur Folge, dass hiermit weder dem Wald, noch den Waldbesitzern noch den Waldnutzern gedient ist. Die Waldbewirtschaftung wird vernachlässigt, die Waldvermehrung nicht gefördert, administrative und juristische Probleme nehmen zu.

Die Forstgesetzgebung in Belgien wurde lange Zeit durch das Forstgesetzbuch von 1854 geregelt. Diese Gesetzgebung war jedoch nur für den öffentlichen Wald gültig. Daneben wurden im Laufe der Jahre eine Reihe weiterer Gesetzgebungen zum Wald erlassen, die nicht zwischen öffentlichem und privatem Wald unterschieden. Hauptsächlich zu erwähnen sind in diesem Zusammenhang:

1. Die Jagdgesetzgebung von 1882, revidiert in 1985.

2. Das Feldgesetzbuch von 1886. Es beinhaltet eine Vielzahl von Regelungen zur Pflanzung von Bäumen und Wäldern auf landwirtschaftlich genutzten Flächen.

3. Das Gesetz von 1931 zur Erhaltung von Naturdenkmalen und Landschaften. Dieses Gesetz schützt bestimmte Wälder auf eine spezifische Weise, revidiert in 1996.

4. Gesetz von 1931 hinsichtlich des Schutzes von privaten Wäldern. Dieses Gesetz regelte außerordentliche Hiebe im Privatwald. Es wurde jedoch nie angewandt.

5. Das Raumordnungsgesetz von 1962: dieses Gesetz regelte die Umwandlung, hauptsächlich für den Privatwald, revidiert in 1998.

6. Die Pachtgesetzgebung von 1969. Sie regelt die Erstaufforstung auf landwirtschaftlichen Flächen.

7. Das Naturschutzgesetz von 1973.

Daneben sind in Belgien noch mehrere kleinere und umfangreichere Gesetze in Kraft, u.a. die Verfassung, die einen wichtigen Einfluss auf die Waldbewirtschaftung haben können.

Es ist deutlich, dass eine derartige Lage Probleme verursacht und sicherlich nicht als ideal angesehen werden kann. Sie ist äußerst nutzerunfreundlich.

In Flandern, eine von drei Hauptregionen in Belgien, ist die Problematik Wald-Natur seit 1970 deutlich geworden. Vorher hat das Thema Natur kaum eine Rolle gespielt. Zwar gab es seit 1957 eine Vielzahl wertvoller staatlicher Naturschutzgebiete, sie wurden jedoch problemlos durch die Forstverwaltung betreut. Übrigens waren in diesen Schutzgebieten kaum Waldelemente enthalten.

Das erste Naturschutzgesetz wurde 1973 erlassen. Zu dieser Zeit gab es noch keine Naturschutzverwaltung als zuständige Behörde. Zuständige Behörde bezüglich der Anwendung dieses Gesetzes war vielmehr die Forstverwaltung. In bezug auf die Forstgesetzgebung standen zwei wichtige Bestimmungen in diesem Gesetz: 
1. Die Ausweisung von Waldschutzgebieten (Waldreservaten, Bann- und Schonwald) wurde ermöglicht.

2. Maßnahmen zur Erhöhung des Naturschutzwertes der Wälder konnten getroffen werden.

Die Forstverwaltung hat diesem Gesetz jedoch kaum Aufmerksamkeit geschenkt. Jedenfalls wurden keine Schutzgebiete ausgewiesen und es wurden auch keine besonderen Bemühungen zur Erhöhung des Naturschutzwertes im Wald unternommen.

Diese passive und negative Einstellung der Forstverwaltung gegenüber dem Naturschutzgesetz hat in hohem Maß zu den Konflikten, die später zwischen den Forst- und Naturschutzbehörden entstanden sind, beigetragen. Eine besondere Folge war die Gründung der Naturschutzverwaltung und die Entziehung der Zuständigkeit der Forstverwaltung für Belange des Naturschutzes.

Ein Höhepunkt in der Auseinandersetzung zwischen der Forst- und Naturschutzverwaltung wurde 1989 erreicht, als die Naturschutzverwaltung fünf sehr wertvolle Wälder als Naturschutzgebiete (Naturreservate) klassifizieren ließ. Auf diese Weise wurde die Entscheidungskompetenz der Forstverwaltung hinsichtlich der Waldflächen stark eingeschränkt.

Inzwischen änderten sich in Flandern die waldbaulichen Einsichten in bezug auf die ökologische Funktion des Waldes wesentlich. Dies kam im neuen flämischen Waldgesetz von 1990 deutlich zum Ausdruck.

\section{2. Ökologische Aspekte des flämischen Waldgesetzes von 1990}

Im flämischen Waldgesetz von 1990 wurde die ökologische Funktion des Waldes zum ersten mal anerkannt und als eine der vielfältigen Waldfunktionen erwähnt. Es war jedoch sehr schwierig, einen Konsens für eine Definition des Begriffes zu finden. Schließlich wurde die ökologische Funktion sehr eng definiert. Dabei wurde lediglich die floristische und faunistische Bedeutung des Waldes beachtet.

Aber gleichzeitig wurden, zerstreut über das Waldgesetz, eine Vielzahl von Dingen betont, die wissenschaftlich gesehen eigentlich zu der ökologischen Funktion gehören. Es handelt sich vor allem um Vorschriften hinsichtlich der Schutzfunktion des Waldes oder des allgemeinen Waldschutzes. Daneben gibt es noch den Abschnitt, der die Waldschutzgebiete betrifft. Im allgemeinen wurde festgestellt, dass die Betonung der ökologischen Funktion des Waldes als eine der wichtigsten positiven Errungenschaften des neuen Gesetzes zu betrachten ist.

Die besonderen Bestimmungen über die rein ökologische Funktion betreffen nur die öffentlichen Wälder. Die Privatwaldbesitzer haben in dieser Hinsicht keinerlei Verpflichtungen. Es gibt eben kaum Fördermittel, um die Privatwaldeigentümer in eine ökologische Richtung zu lenken. Immerhin sind die Fördermittel für den Anbau von autochthonen Laubbaumarten wesentlich höher als jene für den Anbau von Exoten wie Fichte, Douglasie, Kiefer und Lärche.

\subsection{Die ökologische Funktion}


Vier Artikel des Waldgesetzes handeln spezifisch von der ökologischen Funktion des Waldes. Diese Artikel wurden im letzen Moment eingeführt und stellen größtenteils nur eine abgeschwächte Kopie der Bestimmungen zu den Waldschutzgebieten dar.

- Die Bestimmungen betreffen nur die öffentlichen Wälder.

- Die Bewirtschaftung der öffentlichen Wälder sollte sich nach folgenden Leitlinien („anzustrebenden Zielen“) ausrichten:

- die Erhaltung oder die Wiederherstellung der natürlichen Flora und Fauna;

- die Förderung von einheimischen oder standortsgerechten Baumarten;

- die Förderung der Naturverjüngung;

- die Förderung der Ungleichaltrigkeit und der Ungleichförmigkeit;

- die Förderung des ökologischen Gleichgewichtes.

- Eine Reihe von Aktivitäten sind verboten, es sei denn dass sie durch die Forsteinrichtung (den Bewirtschaftungsplan) ausdrücklich vorgesehen oder von der Forstverwaltung genehmigt wurden.

Genannte Bestimmungen sind nicht gerade konkret. So ist der Begriff Leitlinien („anzustrebende Ziele") unklar: in welchen Maß müssen die verschiedenen Vorgaben umgesetzt werden? Im Bewirtschaftungsplan muss wohl angegeben werden, in welchem Maß die ökologische Funktion berücksichtigt wird - auch die Privatwaldbesitzer sollen dies tun - aber in welcher Form und Weise, das wie, bleibt offen. Ein wichtiger Diskussionspunkt betrifft die Bedeutung von ${ }_{n}$ standortsgerechten Baumarten". Dieser Begriff kann nicht definiert werden, aber ist sicher viel weiter gefasst als der Begriff „einheimische Baumarten“. Er hat keine absolute Bedeutung und sein Inhalt kann je nach zeitlicher Strömung variieren. So gab es 1990 zum Beispiel keine Diskussion über die Amerikanische Eiche, während diese Baumart im Jahr 2000 in öffentlichen Wäldern kaum mehr geduldet wird.

\subsection{Die Schutzfunktion}

Im Waldgesetz von 1990 spricht man zum erstenmal von Schutzwäldern. Hierbei wurde vor allem an folgende Funktionen gedacht:

- Wasserschutzgebiete;

- erosionsempfindliche Gebiete;

- Wälder, die für die Regelung des Wasserregimes, des Klimas und der Trinkwassergewinnung von Bedeutung sind;

- Puffergebiete in der Nähe von umweltbelastenden Betrieben.

Bemerkenswerte Bestimmungen sind folgende:

- Schutzwälder werden durch die flämische Regierung ausgewiesen. Die Einwilligung des Besitzers ist nicht erforderlich.

- Eine Entschädigung in Abhängigkeit von den auferlegten Beschränkungen des Eigentumsrechtes wird dem Besitzer zuerkannt.

- Neue Wälder können angebaut werden um als Schutzwald zu dienen. 
1999 waren in Flandern noch keine Schutzwälder ausgewiesen. Ein vorbereitendes Studium, um Kriterien für die Selektion von Schutzwäldern festzulegen, wurde jedoch bereits abgeschlossen.

\subsection{Die Waldschutzgebiete (Waldreservate, Bann- und Schonwald)}

Während der Vorbereitung des Waldgesetzes wurde der Inhalt dieses Abschnittes intensiv diskutiert. Hinsichtlich Definition, Zielsetzungen und der Anzahl der Gebiete gab es große Meinungsunterschiede. Aber vor allem wurde die Funktion der Wälder in Naturschutzgebieten hartnäckig diskutiert.

Zuerst wurden die Artikel über die Waldschutzgebiete, die im Naturschutzgesetz von 1973 aufgenommen wurden, aufgehoben. Die Zuständigkeit für die Waldschutzgebiete wurde dadurch wieder offiziell der Forstverwaltung übertragen.

Bei der Zielsetzung der Waldschutzgebiete waren zwei Standpunkte zu unterscheiden:

- die Betonung der wissenschaftlichen Funktion, wie dies in den mitteleuropäischen Ländern traditionell gehandhabt wurde;

- die Betonung des ökologischen Wertes der Waldschutzgebiete.

Im Jahre 1990 wurde endlich beschlossen, dass für Waldschutzgebiete besondere Schutz- und Behandlungsrichtlinien festgelegt werden müssen, weil sie vor allem eine wissenschaftliche Funktion für die Forstwirtschaft zu erfüllen haben.

Laut Gesetz können die Waldschutzgebiete eine doppelte Zielsetzung haben:

- das Wachstum und die Entwicklung des Waldes bleiben völlig ungestört (Totalreservate, Bannwälder oder "strikte Waldreservate")

- die Entwicklung typischer Waldpflanzengemeinschaften, Waldbestandstypen und Wachstumsformen wird angestrebt (Schonwälder oder "gerichtetete Waldreservate").

Ein Problem ist, dass nur noch sehr wenige Wälder überhaupt noch einen ausreichenden Grad an Natürlichkeit aufweisen und sich damit für die Ausweisung als Waldschutzgebiet eignen. Politische Vorgabe ist es ausdrücklich, nur dann Bannwälder anzuweisen, wenn der Wald größtenteils aus einheimischen Baumarten besteht oder wenn im Rahmen eines kurzen Übergangszeitraums mit Hilfe von Pflegemaßnahmen die nicht erwünschten Baumarten eliminiert werden können. Bei der Bewirtschaftung der Schonwälder gilt es hingegen, die Schutzziele zu beachten und umzusetzen. Diese sind identisch mit den Leitlinien hinsichtlich der ökologischen Funktion der Wälder. Der Ausdruck "Schutzziele" ist hierbei verbindlicher als der Ausdruck "Leitlinien", der mehr Gestaltungsspielraum lässt.

Waldschutzgebiete können, im Gegensatz zu Schutzwäldern, nur ausgewiesen werden unter der Voraussetzung dass der Besitzer einverstanden ist. Selbstverständlich können auch Privatwälder ausgewiesen werden. Eine Entschädigung auf Basis des Einkommensverlustes ist gleichfalls vorgesehen. Alle Waldschutzgebiete, auch private, werden von der Forstverwaltung betreut. Ein Bewirtschaftungsplan (eine Behandlungsrichtlinie) muss erarbeitet werden. Eine 
Reihe von Verbotsbestimmungen sind gültig innerhalb des Schutzgebiets, aber können mittels des Bewirtschaftungsplans oder infolge einer Genehmigung der Forstverwaltung aufgehoben werden. Aber auch in der unmittelbaren Umgebung von Waldschutzgebieten können Maßnahmen zur Beseitigung von negativen Einflüssen getroffen werden. Hierfür können Bewirtschaftungsverträge im Rahmen des Vertragsnaturschutzes abgeschlossen werden. Sie betreffen hauptsächlich die Verwendung von Bioziden, die Regelung der Wasserstände und die Form der Flächennutzung.

Die Diskussion hinsichtlich des Verhältnisses zwischen Waldschutzgebieten und Naturschutzgebieten konnte nicht befriedigend gelöst werden. Die Naturschutzverwaltung hatte die Absicht, Wald durch den Status eines Naturschutzgebietes der Forstverwaltung zu entziehen. Diese Haltung befand sich jedoch im Widerspruch zu dem allgemeinen Prinzip dass „alle Wälder unter die Bestimmungen des Waldgesetzes fallen“.

Es wurde im folgenden beschlossen, dass:

- die Wälder, die bereits den Status eines Naturschutzgebietes erworben haben, praktisch völlig in den Zuständigkeitsbereich der Naturschutzverwaltung fallen;

- für Wälder in Naturschutzgebieten gemäss Bestimmung des Naturschutzgesetzes ein Bewirtschaftungsplan (eine Behandlungsrichtlinie) erarbeitet werden muss.

Die Frage, welche Wälder in Zukunft den Status eines Naturschutzgebietes und welche Wälder den Status eines Waldschutzgebietes erhalten sollten, konnte nicht beantwortet werden. Als Richtlinie wurde formuliert, dass diejenigen Gebiete in denen die Waldfläche deutlich dominiert als Waldschutzgebiete klassifiziert werden sollen. Andererseits sollen Gebiete in denen eine erhebliche unbewaldete Fläche vorhanden ist, den Status eines Naturschutzgebietes erhalten. In der Praxis blieb die Diskussion jedoch bestehen. Öfentliche Wälder wurden zwar nicht mehr als Naturschutzgebiete klassifiziert, wohl aber Privatwälder.

\subsection{Allgemeiner Schutz des Waldökosystems}

Das Waldgesetz von 1990 strebt im allgemeinen die „Erhaltung" und den „Schutz" des Waldes an.

Eine erste Gruppe von Regelungen bezieht sich auf die Walderhaltung:

1. Die Umwandlung ist prinzipiell verboten. Sie ist nur zulässig im Rahmen der Regelungen des Gesetzes über die Räumliche Ordnung von 1962, welches beinhaltet, dass eine Genehmigung der Gemeinde (des Landratsamtes) erforderlich ist. Das Waldgesetz hat diese Regelung, die in der Vergangenheit eher ungünstig war, nicht ändern können. Es wurde nur hinzugefügt, dass die Forstverwaltung zu jedem Antrag auf Umwandlung ihre Stellungnahme abgeben muss.

2. Die Veräusserung von öffentlichen Wäldern ist strengen Regeln unterworfen. Für Staatswald ist eine Verordnung, für sonstige Waldbesitzformen eine Genehmigung der flämischen Regierung erforderlich. 
3. Die Probleme der Waldzerstückelung werden durch die Aufstellung des Bewirtschaftungsplans in Grenzen gehalten.

4. Die Forstbehörde kann in bestimmten Fällen Waldbesitzer enteignen: für Zwecke der Arrondierung, für den Erwerb bedrohter Wälder sowie den Erwerb von Waldschutzgebieten und Schutzwäldern.

Ein anderer Abschnitt des Gesetzes dient dem Zweck des Waldökosystemschutzes:

1. Eine Vielzahl von Verbotsbestimmungen sind sowohl für den öffentlichen Wald als auch für den Privatwald gültig.

2. Änderungen des physikalischen Zustandes sind nur mit einer Genehmigung zulässig. Weitere Erlasse hierzu müssen von der flämischen Regierung noch herausgegeben werden, dies ist bisher noch nicht geschehen.

3. Schließlich gibt es Vorschriften zur Vermeidung und Bekämpfung von Waldbränden sowie zur Sanierung waldbrandgeschädigter Flächen.

\subsection{Allgemeine Bestimmungen zur Unterstützung der ökologischen Funktion}

Eine Hauptzielsetzung des Waldgesetzes war die Erhöhung des Naturschutzwertes der Wälder. Darum wurden nicht nur spezifische Abschnitte eingebaut, sondern auch Bestimmungen, die an diese ökologischen Zielsetzungen des Gesetzes erinnern müssen, wurden in anderen Abschnitten aufgenommen:

- In der allgemeinen Zielsetzung des Gesetzes wird deutlich formuliert, dass die Erhaltung und der Schutz des Waldes Priorität besitzen.

- In der Definition des Begriffes Wald wird speziell auf die waldspezifische Flora und Fauna verwiesen.

- Bei der ökonomischen Funktion wird betont, dass der Wald als ein erneuerbarer Rohstoff betrachtet werden muss.

- Die Erholung im Wald ist nur gestattet unter der Voraussetzung dass die Erhaltung des Waldes gesichert ist.

- Die Forsteinrichtung muss dem Geist des Waldgesetzes entsprechen und die ökologische Funktion respektieren.

\subsection{Gutachten/ Stellungnahme des Flämischen Hohen Rates für den Naturschutz}

Das Waldgesetz von 1990 sieht vor, dass der Flämische Hohe Rat für Naturschutz seine Stellungnahme abgeben bzw. ein Gutachten erstellen muss zu bestimmten ökologischen 
Belangen im Wald. Diese Belange sind jedoch begrenzt auf: langfristige Planung, Kriterien für Schutzwälder, Ausweisung von Waldschutzgebieten, von der Schutzverordnung abweichende staatliche Eingriffe in Waldschutzgebiete.

\section{Direkte Bestimmungen des Naturschutzgesetzes von 1997 zum Wald}

Im Naturschutzgesetz von 1997 wurden einige Artikel eingefügt die zur Änderung des Waldgesetzes von 1990 führen. Das direkte Ziel dieser Artikel ist die Erhöhung des Naturschutzwertes der Wälder. Eine indirekte Folge ist selbstverständlich, dass die Naturschutzverwaltung einen grösseren Einfluss auf die Forstpolitik und die forstliche Zuständigkeitskompetenz ausübt.

1. Das Naturschutzgesetz enthält die Regeln für die Einführung eines „Flämischen Ökologischen Netzwerkes" (VEN). Die Naturschutzfunktion ist prioritäres Ziel in diesen Gebieten. In diesem Netzwerk werden natürlich auch viele Wälder gelegen sein. Es ist zur Zeit jedoch unklar, welche Verpflichtungen sich hieraus für die Waldbesitzer dieser Gebiete ergeben werden.

Durch das Gesetz wird die Forderung nach einem Bewirtschaftungsplan (einer Behandlungsrichtlinie) für öffentliche Wälder in diesem Flämischen Ökologischen Netzwerk betont:

- die Naturschutzverwaltung muss hierzu eine Stellungnahme abgeben;

- die Naturschutzverwaltung muss regelmäßig über die Durchführung und Umsetzung des Bewirtschaftungsplans informiert werden;

- Die Behandlungsrichtlinie muss wenigstens die Daten enthalten, die für Bewirtschaftungspläne für Waldschutzgebiete vorgesehen sind. Dies bedeutet dass sie detaillierte Daten hinsichtlich der ökologischen Zielsetzung enthalten muss.

2. Für die Privatwälder, die im VEN gelegen sind, muss der Bewirtschaftungsplan deutlich machen, welche Pflege- und Entwicklungsmaßnahmen zur Erreichung der anzustrebenden ökologischen Zielsetzungen festgelegt werden.

Laut Waldgesetz gab es diese Verpflichtungen ursprünglich nur für den öffentlichen Wald.

3. Die Regulierung hinsichtlich der Aufstellung von Bewirtschaftungsplänen für in Naturschutzgebieten gelegene Wälder wird geändert:

- Der Bewirtschaftungsplan wird gemäss der Bestimmungen des Naturschutzgesetzes von 1997 aufgestellt.

- Die Forstverwaltung muss inre Stellungnahme abgeben.

Hierdurch wird die Regulierung klarer, besonders hinsichtlich des Verhältnisses zwischen der Forstverwaltung und der Naturschutzverwaltung. Die Forstverwaltung hat eine Informationsfunktion.

4. Umwandlung in Naturschutzgebieten wird vereinfacht 
Umwandlung in Naturschutzgebieten ist möglich, vorausgesetzt, dass sie Teil des Bewirtschaftungsplans ist. Die Umwandlung muss nicht mehr von der Gemeinde genehmigt werden. Sie muss nur noch gemeldet werden.

5. Die Regelungen für Umwandlung (außerhalb von Naturschutzgebieten) werden viel strikter. Eine Genehmigung für eine Umwandlung darf nur noch für Zwecke des öffentlichen Allgemeinwohls erteilt werden. Übrigens muss die flämische Regierung die Kriterien für die Ersatzaufforstung (Ausgleichsmaßnahmen) bei einer Umwandlung festlegen.

6. Die flämische Regierung kann für das Festlegen von Maßnahmen, die die natürliche Entwicklung des Waldes zum Ziele haben, Fördermittel vergeben.

Derartige Maßnahmen waren im Waldgesetz nicht explizit vorgesehen, aber wohl möglich. Ähnliche allgemeine Maßnahmen sind auch im Naturschutzgesetz vorgesehen.

7. Stellungnahme zur Erstaufforstung von in "Naturschutzgebieten" gelegenen Flächen

Das Waldgesetz bestimmte, dass für die Erstaufforstung einer Vielzahl von Naturschutzgebieten eine Stellungnahme der Naturschutzverwaltung erforderlich war. Das Naturschutzgesetz weitet diese Bestimmung aus auf sonstige Flächen, die für den Naturschutz von Bedeutung sind.

Der Naturschutz will verhüten, dass Flächen mit einem hohen Naturschutzwert aufgeforstet werden und dadurch ihren Naturschutzwert verlieren.

8. Die flämische Regierung muss Kriterien für ökologisch zu verantwortende Erstaufforstung und Waldvermehrung festlegen.

Das Gesetz über die räumliche Ordnung sieht vor, dass 10.000 ha neue Wälder auf eine ökologisch sinnvolle Weise angebaut werden müssen. Dieser Begriff war jedoch nicht präzise definiert. Waldvermehrung in Naturschutzgebieten wird auch heftig diskutiert. Diese Richtlinien sollen hier Abhilfe schaffen.

\section{Indirekte Bestimmungen des Naturschutzgesetzes von 1999 zum Wald}

Das Naturschutzgesetz sieht neben direkten Änderungen des Waldgesetzes auch eine Reihe von Bestimmungen vor, die ein breites Anwendungsgebiet haben, weil sie im allgemeinen die Natur fördern möchten. Es ist klar, dass auch die Waldreglementierung in hohem Maß hiervon bestimmt wird.

Das Naturschutzgesetz formuliert in einem ersten Teil die allgemeinen Zielsetzungen, einige allgemeinen Bestimmungen und Maßnahmen zur Förderung des Naturschutzes. Im zweiten Teil wird eine besondere Reglementierung zur Förderung einer an Schutzgebieten ausgerichteten Naturschutzpolitik ausgearbeitet. Daraufhin kommen eine Reihe von allgemeinen Maßnahmen zum Schutz der natürlichen Umwelt. Zum Schluss gibt es auch spezifische Bestimmungen zum Schutz von Pflanzen- und Tierarten. Alle diese Maßnahmen betreffen auch mehr oder wenig den Wald und die Waldfläche. Ihr Einfluss ist zu diesem Zeitpunkt jedoch noch nicht hinreichend klar. Hierfür gibt es zwei Gründe: 
- Zum einen sind viele Bestimmungen des Naturschutzgesetzes allgemeine Bestimmungen, die wenig konkret sind und sehr unterschiedlich interpretiert werden;

- zum anderen müssen zahlreiche Bestimmungen des Naturschutzgesetzes noch im Rahmen von Verordnungen der flämischen Regierung konkretisiert werden.

\subsection{Allgemeine Bestimmungen des Naturschutzgesetzes}

Das Naturschutzgesetz enthält eine Vielzahl "horizontaler Maßnahmen“. Dies sind Maßnahmen die auf das ganze flämische Gebiet, also auch auf die Wälder, anwendbar sind. Daneben gibt es viele Maßnahmen die nur auf bestimmte Gebiete anwendbar sind.

1. Gemäß der gesetzlichen Definition gehören die Wälder zum Begriff "Natur“.

Dies bedeutet, dass Wälder unter das Naturschutzgesetz fallen. Die hieraus entstehenden Konsequenzen sind jedoch noch nicht offensichtlich. Das Naturschutzgesetz gibt auch eine Definition und eine Reihe von Bestimmungen für den Begriff Landschaftsstrukturelemente („kleine Landschaftselemente“). Hierzu gehören auch die kleinflächigen Waldstrukturen (Baumgruppen, „kleinen Wälder"). Eine Definition für kleinflächige Waldstrukturen wird jedoch nirgendwo gegeben, weder im Waldgesetz noch im Naturschutzgesetz.

2. Die flämische Regierung ist dazu befugt, für das Territorium Flanderns sämtliche erforderliche Vorschriften und Maßnahmen zur Sicherung einer Umweltqualität, die für die Erhaltung der Natur erforderlich sind, zu treffen und das "Stand-Still“ Prinzip sowohl in bezug auf die Qualität als auch auf die Quantität der Natur anzuwenden. Dies bedeutet, dass die Qualität und die Quantität der Umwelt, einschließlich der Natur, nicht vermindert werden darf. Dieses Prinzip ist ebenfalls auf die Waldbewirtschaftung anwendbar. (Das Prinzip des Nutzungsrechts ist jedoch Rahmenbedingung, s. folgende.)

3. Die flämische Regierung ist auch dafür zuständig, nötige Vorschriften für den Naturschutz zu erlassen. Dies betrifft sowohl Vorschriften zugunsten der bestehenden Natur als auch für die Erhaltung der natürlichen Umwelt. (Aber auch hier ist das Prinzip des Nutzungsrechts gültig.)

Die Tragweite dieser Bestimmung ist jedoch besonders unkonkret. Darum wurde versucht, mittels einer Verordnung der flämischen Regierung mehr Deutlichkeit zu schaffen. Diese Verordnung legt die Voraussetzungen für Änderungen an der bestehenden Vegetation und an Landschaftsstrukturelementen fest. Sie wurde ihrerseits wiederum durch einen Rundbrief näher erläutert und sieht drei Möglichkeiten vor:

1. Das Ändern einer Vielzahl von Landschaftsstrukturelementen und Vegetationsformen ist verboten. In den Wäldern sind dies vor allem hohle Wege, Quellen und eine Reihe von Waldgesellschaften der Sümpfe und Küsten. Diese Bereiche haben also wenig oder gar nichts mit einer produktionsorientierten Forstwirtschaft zu tun. Übrigens regelt die Verordnung, dass die Verbotsbestimmungen nicht gültig sind wenn es einen genehmigten Bewirtschaftungsplan gibt oder es um normale Pflegemaßnahmen geht. 
2. Für die Änderung der Vegetation in allerlei Naturschutzgebieten (einschließlich der Wälder) ist eine Genehmigung der Naturschutzverwaltung erforderlich. Der Begriff „Änderung" ist selbstverständlich nicht näher definiert, aber spezifisch wird u.a. auf Folgendes hingewiesen:

- das Vernichten, Beschädigen oder Abtöten der Vegetation;

- die künstliche Bepflanzung von Vegetationsflächen;

- die vollständige oder teilweise Umwandlung kleiner Waldflächen;

- die Änderung des Wasserhaushalts.

Für alle Aktivitäten, die in einem genehmigten Bewirtschaftungsplan vorgesehen sind, wie auch für normale Pflegemaßnahmen besteht keine Genehmigungspflicht. Eine genaue Definition für alle diese Begriffe gibt es jedoch nicht.

Diese Bestimmung hat also nur eine Bedeutung für diejenigen Wälder, für die kein genehmigter Bewirtschaftungsplan besteht.

3. In einer Vielzahl von Fällen müssen die Änderungen nur der Gemeinde gemeldet werden, so z.B. die Umwandlung von Laubwald in Agrargebieten. Diese Bestimmung scheint im Widerspruch zum Waldgesetz zu stehen, in dem für die Umwandlung von derartigen Wäldern zuerst eine Meldung an die Landwirtschafts- und die Forstverwaltung erforderlich ist.

4. Zum Schutz der Landwirtschaft (und gleichzeitig der Forstwirtschaft) wurde das sehr wichtige Prinzip des "Nutzungsrechtes" im Naturschutzgesetz eingeführt. Dies bedeutet, dass die oben erwähnten Vorschriften (Punkte 2 und 3) keine Einschränkung der Aktivitäten, die normalerweise zur Ausführung des der räumlichen Ordinung entsprechenden Flächennutzungsplans nötig sind, beinhalten dürfen. Dies wiederum hat zur Folge, dass die Forstwirtschaft wohl bestimmten Rahmenbedingungen unterworfen, aber nicht unmöglich gemacht werden kann.

Die Frage ist nun selbstverständlich, was dies konkret bedeutet und wie alle diese Bestimmungen miteinander zu vereinbaren sind.

5. Eine theoretisch sehr wichtige Vorgabe des Naturschutzgesetzes betrift die "Sorgepflicht". " Ein jeder der Handlungen verrichtet oder hierzu Auftrag erteilt und der weiss oder billigerweise vermuten kann, dass die Naturelemente in der unmittelbaren Nähe dadurch vernichtet oder schwer geschädigt werden können, ist dazu verpflichtet, alle Maßnahmen zu treffen, die billigerweise von inm zur Verhütung, Beschränkung oder Wiederherstellung verlangt werden können".

Diese horizontale Maßnahme ist einer der Kernpunkte des neuen Gesetzes. Es ist jedoch wiederum nicht klar, was eine derartige Bestimmung für den Forstsektor bedeutet.

6. Das Naturschutzgesetz wendet auch das "Integrationsprinzip" der europäischen Umweltpolitik an. Eine Behörde, die eine Genehmigung erteilen muss, soll geeignete Auflagen festlegen, die vermeidbare Schäden an der Natur verhindern können. Dieser Artikel ist wichtig für die Erteilung 
einer Hiebsgenehmigung, wobei selbstverständlich die Frage aufkommt: was ist vermeidbarer Schaden? Schädigt nicht jeder Hieb die Natur?

7. Der Flämische Hohe Rat für den Naturschutz, der ein Gutachten über die "Natur" erstellen muss, muss demnach auch regelmäßig ein Gutachten über den Wald als Element der Natur erstellen. Wie bereits erwähnt, sieht auch das Waldgesetz vor, dass der Flämische Hohe Rat für den Bereich Naturschutz ein umfassendes Gutachten erstellen muss.

8. Das Institut für Naturschutz muss regelmäßig einen "Naturzustandsbericht" verfassen. Dieses Gutachten muss selbstverständlich auch über die Bewertung des Naturzustands in Wäldern, die zu erwartende Entwicklung sowie die Evaluation der vergangenen Politik informieren.

Das Gesetz sieht auch vor, dass die Behörden, also auch die Forstverwaltung, dem Institut sämtliche Informationen, die zur Erstellung des Naturzustandsberichts nützlich sein können, zur Verfügung stellen müssen.

\subsection{Die schutzgebietsausgerichtete Politik des Naturschutzes}

Das Gesetz sieht drei verschiedene Kategorien vor:

- Das Flämische Ökologische Netzwerk (VEN). Dies ist ein zusammenhängendes Gebiet, in dem eine spezifische Politik in bezug auf die Natur geführt wird. Ungefähr $10 \%$ der gesamten flämischen Landflächen müssen diesem Netzwerk angehören (125.000 ha).

- Das "Integrale Verwebungs- und Unterstützungs-Netzwerk" (IVON). Dies ist eine Gesamtheit von Gebieten, in denen die Behörde für die Erhaltung des vorhandenen Naturschutzwertes Sorge trägt sowie Maßnahmen zur Unterstützung des Naturschutzwertes und der Biodiversität durchführen kann. 150.000 ha sollen auf diese Weise ausgewiesen werden.

- Naturschutzgebiete sind Gebiete die für die Erhaltung und die Entwicklung der Natur oder der natürlichen Umwelt wichtig sind. In diesen Schutzgebieten wird, mittels einer geeigneten Bewirtschaftung, ein Naturschutz-Leitbild („anzustrebendes Naturschutzziel“) erhalten oder entwickelt.

1. Die Bestimmungen des Flämischen Ökologischen Netzwerks sind für den Forstsektor, insbesondere für die Privatwaldbesitzer, von großer Bedeutung.

Das VEN wird seinerseits in zwei Kategorien aufgeteilt:

- Naturschutzgebiete bzw. "Große Einheiten Natur" (GEN): wertvolle Naturschutzflächen mit einem hohen aktuellen Naturschutzwert;

- Naturschutzentwicklungsgebiete bzw. "Große Einheiten Natur in Entwicklung" (GENO): Gebiete mit einem hohen potentiellen Naturschutzwert.

In beiden Gebieten ist die Naturschutzfunktion dominant, freilich etwas weniger in den GENO's. Spätestens im Jahre 2008 müssen für diese Gebiete Behandlungsrichtlinien („Naturschutzpläne") erstellt sein.

Bei der Ausweisung dieser Schutzgebiete hat der Flämische Hohe Forstrat nur eine beratende Funktion. Praktisch alle Wälder können in diesem Netzwerk aufgenommen werden. 
Es ist für die Eigentümer nicht ersichtlich, welche Folgen sich für die in diesem Netzwerk klassifizierten Wälder ergeben. Wie bereits erwähnt, sieht das Naturschutzgesetz wohl einige direkte Bestimmungen, sowohl für die öffentlichen Waldbesitzer als auch für die Privatwaldbesitzer vor:

- Beide Gruppen müssen im Bewirtschaftungsplan die Maßnahmen, die zur Realisierung der ökologischen Zielsetzungen nötig sind, eindeutig benennen.

- Die Forsteinrichtungswerke müssen den noch zu erstellenden Behandlungsrichtlinien entsprechen.

Übrigens fordert das Naturschutzgesetz weitere konkrete Maßnahmen:

- eine naturnahe Forstwirtschaft sowie die Ausweisung von Waldschutzgebieten muss gefördert werden;

- der Wasserhaushalt muss auf eine hohe Naturschutzqualität abgestimmt werden;

- das Verbot, Pestizide (Schädlingsbekämpfungsmittel) einzusetzen;

- das Verbot, die Vegetation zu verändern, es sei denn dass dies in einem genehmigten Bewirtschaftungsplan vorgesehen ist.

Deswegen fürchtet der Waldbesitzer, dass die ökonomische Funktion seines Waldes stark eingeschränkt werden wird. Viel hängt davon $a b$, was als naturnahe Forstwirtschaft angesehen wird. Wird sie auf freiwilliger Basis durchgeführt, mit Hilfe von Fördermitteln oder Vertragsnaturschutz? Oder umfasst die Förderung auch obligatorische Maßnahmen? Und können diese durch die Behandlungsrichtlinie auferlegt werden? Und was bedeutet in diesem Fall das Prinzip des Nutzungsrechts?

In den Gebieten des IVON haben einige zusätzliche Bestimmungen eine große Bedeutung:

- die Maßnahmen dürfen die Waldnutzung nicht regeln, es sei denn mittels des Instruments des Vertragsnaturschutzes;

- eine Behandlungsrichtlinie muss erstellt werden;

- die Erhaltung eines für die Natur günstigen Wasserhaushalts ist eine allgemeine Zielsetzung; die physische Umwelt darf wiederhergestellt werden, z.B. das Wiedervernässen von trockengelegten Gebieten.

Die ökonomische Funktion des Waldes ist hier deutlich weniger eingeschränkt, zumindest wenn die Vorgaben der Behandlungsrichtlinie und die Maßnahmen, die die Behörden durchführen sollen, den Bestimmungen des Gesetzes entsprechen.

3. Wie bereits erwähnt, hat das Naturschutzgesetz auf eine direkte Weise Bestimmungen für in Naturschutzgebieten gelegene Wälder eingeführt:

- die Forsteinrichtung wird nach den Vorgaben des Naturschutzes ausgerichtet;

- die Umwandlung wird erleichtert.

Wälder können sowohl den Status eines Waldschutzgebietes als auch jenen eines Naturschutzgebietes erhalten. In Naturschutzgebieten wird mittels einer geeigneten 
Bewirtschaftung ein bestimmter Schutzzweck angestrebt. Die Fläche zu belassen, „nichts zu tun" ist möglich, aber nicht üblich.

Für Naturschutzgebiete wird der Bewirtschaftungsplan von der Naturschutzverwaltung erstellt.

Die Bewirtschaftung von Naturschutzgebieten geschieht durch einen Beamten der Naturschutzverwaltung.

Für Naturschutzgebiete besteht das Vorkaufsrecht in der unmittelbaren Umgebung.

\subsection{Allgemeine Maßnahmen zum Schutz der Umwelt}

Das Naturschutzgesetz sieht eine Reihe von allgemeinen Maßnahmen zum Schutz der natürlichen Umwelt vor, die z.T. auch für den Forstsektor von Bedeutung sind.

\section{Der Grunderwerb}

- Die flämische Regierung (öffentliche Hand) hat unter bestimmten Voraussetzungen das Vorkaufsrecht, u.a. für im VEN gelegene Wälder, für in Naturschutzgebieten gelegene Wälder, für ihre unmittelbare Umgebung, für in und rund um das IVON gelegene Wälder und in spezifischen "Naturschutzentwicklungsprojekten". Dieses Vorkaufsrecht ist jedoch an bestimmte Voraussetzungen gebunden und gilt nicht im Falle eines Verkaufs an Mitglieder von anerkannten forstlichen Zusammenschlüssen (Waldgruppierungen).

- Anerkannte landbewirtschaftende Naturschutzverbände haben in obenerwähnten Gebieten auch ein Vorkaufsrecht, aber dieses Recht ist beschränkt auf Immobilien, die sie zur Miete oder Pacht innehaben.

- Die flämische Regierung und die Gemeinden können aus Naturschutzgründen und zum Wohle der Allgemeinheit Immobilien durch Enteignung erwerben.

- Der Besitzer eines im Flämischen Ökologischen Netzwerk aufgenommenen Waldes kann fordern, dass die flämische Regierung diesen Wald erwirbt, wenn er meint, dass der Wert dieses Waldes durch die Ausweisung stark gemindert wird.

\section{Vertragsnaturschutz}

Die flämische Regierung kann mit den Landnutzern für Zwecke des Naturschutzes Bewirtschaftungsverträge abschließen. Die Exotenbewirtschaftung, wie die Bekämpfung von Prunus serotina, ist ein typisches Beispiel für den Forstsektor.

Im allgemeinen können Bewirtschaftungsverträge für alle Wälder abgeschlossen werden, inbesondere aber für im VEN oder im IVON gelegene Wälder.

\section{Naturschutzprojekte}

Besondere Projekte des Naturschutzes haben die optimale Einrichtung eines Gebietes zur Erhaltung, Wiederherstellung oder Entwicklung der Natur und der natürlichen Umwelt zum Ziel. Derartige Projekte sind nur möglich, wenn zumindest $90 \%$ der Fläche im VEN oder in Waldgebieten gelegen sind.

Die weiteren Bestimmungen dieses Artikels verweisen nicht explizit auf Elemente der Waldbewirtschaftung, aber wohl auf den Wasserhaushalt. Es ist wiederum nicht deutlich, welchen Einfluss dlese Bestimmungen auf den Forstsektor haben kőnnen. 
4. Behandlungsrichtlinien bzw. Pflege- und Entwicklungspläne

Wie bereits erwähnt müssen für alle Gebiete (einschließlich der Wälder) im VEN oder im IVON Behandlungsrichtlinien erstellt werden. Der Inhalt dieser Pläne ist zur Zeit nicht bekannt, aber die Gebiete müssen trotzdem bereits ausgewiesen werden. Es ist also nicht bekannt, welche Verpflichtungen diesen Gebieten auferlegt werden. Normalerweise sollen die ökologischen Funktionen dieser Gebiete betont werden, was impliziert, dass die ökonomischen Funktionen des Waldes eingeschränkt werden.

Vermutlich wird viel von der Interpretation des Begriffes „Förderung der naturnahen Waldbewirtschaftung" abhängen.

\subsection{Zusätzliche Bestimmungen}

1. Zum Schutz von Pflanzen- und Tierarten und ihrer Lebensgemeinschaften kann die flämische Regierung Maßnahmen treffen, um Populationen von Arten aufrechtzuerhalten, wiederherzustellen oder zu entwickeln. Diese Maßnahmen können überall (horizontale Maßnahmen) oder für bestimmte Gebiete oder Habitate getroffen werden.

Diese Bestimmungen werden jedoch durch das bekannte aber undeutliche Prinzip des Nutzungsrechts eingeschränkt.

2. Ins Gewicht fallende Waldschäden, die durch geschützte Tierarten verursacht wurden, werden durch einen speziellen Fonds entschädigt.

3. Die Beamten der Forstverwaltung sind befugt, im Falle von Naturschutzdelikten zu ermitteln.

4. Die flämische Regierung kann, zur Verhütung von wichtigen Waldschäden, von den Verbotsbestimmungen dieses Gesetzes abweichen.

5. Auf einem Uferstreifen von $6 \mathrm{~m}$ entlang der Fließgewässer bleibt es verboten, Nadelhölzer zu pflanzen oder ihre Sämlinge wachsen zu lassen.

6. Die Forsteinrichtung von in Landschaftsschutzgebieten gelegenen Wäldern muss dem Pflegemaßnahme- und Entwicklungsplan entsprechen. Für in Landschaftsschutzgebieten und in Naturschutzgebieten gelegene Wälder wird die Forsteinrichtung am Naturschutzgesetz ausgerichtet.

\section{Neue ökologische Bestimmungen des geänderten Waldgesetzes von 1999}

Das Waldgesetz von 1990 wurde 1999 in Teilen erheblich geändert. Eine Vielzahl von diesen Modifizierungen verstärken oder verändern den Naturschutzwert des Waldes in bedeutendem Umfang. 


\subsection{Die ökologische Waldfunktion}

1. Die ökologische Funktion wird völlig neu definiert. Sie umfasst jetzt u.a.:

- die Förderung autochthoner Baum-oder Straucharten;

- die Förderung der Bioautomation (biologisch funktionierender Prozesse);

- die Förderung einer vielfältigen Waldstruktur, u.a. durch Ungleichaltrigkeit und Ungleichförmigkeit und durch das Streben nach ausreichenden Anteilen von Alt- und Totholz;

- eine geeignete Bewirtschaftung aller Naturelemente und aller landschaftsökologisch und kulturhistorisch wertvoller Elemente;

- die Bewirtschaftung zugunsten der Erhaltung, der Entwicklung oder der Wiederherstellung der biologischen Vielfalt, zugunsten Populationen von seltenen Arten oder Subarten und zugunsten der Erhaltung, der Entwicklung oder der Wiederherstellung von natürlichen oder z.T. natürlichen Habitaten oder Ökosystemen;

- die Erhaltung oder die Wiederherstellung des natürlichen Wasserhaushalts;

- den Schutz gegen alle nachteiligen Einflüsse von außen.

2. Bei der Bewirtschaftung öffentlicher Wälder muss stets die oben definierte ökologische Funktion berücksichtigt werden.

3. Die Verbotsbestimmungen für öffentliche Wäldern werden strikter. Im Prinzip ist es verboten, den Wasserhaushalt zu ändern, Pestizide oder Dünger anzuwenden.

\subsection{Die Waldschutzgebiete}

1. Der Begriff Waldschutzgebiet wurde neu definiert. In Waldschutzgebieten ist nicht nur die wissenschaftliche Funktion von Bedeutung, sondern in gleichem Maße auch die ökologische Funktion. Waldschutzgebiete können wegen ihrer ökologischen Bedeutung ausgewiesen werden.

2. Die Bewirtschaftung eines Waldschutzgebiets erfolgt nicht mehr in allen Fällen durch die Forstverwaltung. Dies ist nur noch der Fall für Waldschutzgebiete im flämischen Staatswald oder für Waldschutzgebiete, die einer Pacht durch die flämische Regierung unterliegen. Andere Besitzer können die Bewirtschaftung der Forstverwaltung übertragen.

3. Die Verbotsbestimmungen werden noch strikter. Im Prinzip ist es verboten zu jagen, Pestizide oder Dünger anzuwenden oder bauliche Anlagen jeder Art zu errichten.

\subsection{Die Bewirtschaftung der Wälder}

1. Die flämische Regierung muss Kriterien für eine nachhaltige Waldbewirtschaftung entwickeln. 
2. Die Forstbetriebe müssen bestimmte Zielsetzungen anstreben:

- die Auffassungen hinsichtlich der Waldbewirtschaftung müssen dem Naturschutz entsprechen;

- sie sollen sich aktiv um die Realisierung der Zielsetzungen und Maßnahmen der Behandlungsrichtlinien bemühen.

3. Die Forsteinrichtung öffentlicher Wälder des Flämischen Ökologischen Netzwerkes muss nicht mehr nach den für Waldschutzgebiete gültigen Regelungen erfolgen.

4. Der Besitzer eines öffentlichen Waldes kann die Bewirtschaftung eines Waldes einem Naturschutzverband anvertrauen, zumindest wenn dieser Wald den Status eines Wald- oder Naturschutzgebietes inne hat.

\subsection{Umwandlung}

Das allgemeine Prinzip des im Naturschutzgesetz von 1997 festgelegten Umwandlungsverbots mit Ausnahme für Zwecke des öffentlichen Gemeinwohls wird von nun an differenzierter gehandhabt:

- Umwandlung auf den als Wohngebiet oder Industriegebiet vorgesehenen und im Rahmen der Bauleitplanung ausgewiesenen Flächen ist erlaubt.

- Die flämische Regierung kann auf begründeten Einzelantrag des Grundstückseigentümers eine Genehmigung zur Umwandlung erteilen.

- Die flämische Regierung muss noch zu konkretisierende Verordnungen zur Umwandlung erlassen.

- Die Voraussetzungen für einen Ausgleich bzw. eine Ersatzaufforstung werden z.T. bereits festgelegt:

- Der Ausgleich muss in gleichem Umfang erfolgen.

- Für in einem anerkannten FFH-Gebiet gelegene Wälder kann der Ausgleich überproportional, aber höchstens um den Faktor 2 erfolgen.

- Der Antragsteller kann über einen Dritten Bürgschaft gewährleisten.

- Ausgleichsmaßnahmen können nur im Freiraum stattfinden.

- Die Forstverwaltung muss eine Stellungnahme zu den vorgesehenen Ausgleichsmaßnahmen abgeben.

- Die Ausgleichsmaßnahmen sind Voraussetzung für die Genehmigung einer Umwandlung.

\subsection{Beratungsrechts des Flämischen Hohen Rates für den Naturschutz}

Das Beratungsrecht des Flämischen Hohen Rates für den Naturschutz wird ausgedehnt: Gutachten und Stellungnahmen zu erforderlichen Fördermitteln für eine natürlichere Waldentwicklung, Ausführungsverordnungen, Entschädigungen für Schutzgebietsflächen, Kriterien für eine nachhaltige Waldbewirtschaftung, Anerkennung von Forstwirtschaftlichen 
Zusammenschlüssen (Waldgruppierungen), Bewertung von Forstwirtschaftlichen

Zusammenschlüssen und Berufsverfahren.

\section{Diskussion}

\section{1. Änderungen der Auffassungen}

Die Vorstellungen über Forstwirtschaft und Naturschutz haben sich im Laufe des vergangenen Jahrhunderts drastisch geändert (Le Master et al., 1996). Die Gesetzgebung hat sich hieran anpassen müssen. In Flandern sind im Verhältnis zwischen Forstwirtschaft und Naturschutz während der letzten Jahrzehnte fünf Abschnitte zu beobachten:

- Die Forstverwaltung schenkt dem Naturschutz im Wald kaum Aufmerksamkeit. Es gibt auch keine Richtlinien (Zeitraum vor 1973).

- Das Naturschutzgesetz von 1973 formuliert die ersten deutlichen Richtlinien hinsichtlich des Naturschutzes in Wäldern: die Ausweisung von Waldschutzgebieten wird ermöglicht und Maßnahmen zur Erhöhung des Naturschutzwertes in Wäldern können ergriffen werden.

Die Forstverwaltung führt jedoch das Vorhaben nicht aus. Dies erklärt größtenteils warum der Konflikt Forstwirtschaft-Naturschutz nachher solche Dimensionen annimmt. Die Diskussion erreicht 1989 ihren Höhepunkt, als die Naturschutzverwaltung fünf wertvolle Wälder als Naturschutzgebiete ausweist.

- Das flämische Waldgesetz von 1990 hat offensichtlich eine Vielzahl ökologischer Akzente. Die Forstpolitik und die Waldbewirtschaftung werden, den internationalen Entwicklungen gemäß, naturnäher.

- Die politische Macht des Naturschutzes wird immer größer und der direkte bzw. indirekte Einfluss des Naturschutzes auf die Forstpolitik wird deutlicher. Das flämische Naturschutzgesetz von 1997 ändert eine Reihe von Bestimmungen des Waldgesetzes von 1990 auf direkte Weise und in hohem Maße. Aber langfristig gesehen sind wahrscheinlich die Bestimmungen, die einen indirekten Einfluss auf den Wald nehmen, von noch größerer Bedeutung.

- Auch innerhalb des Waldsektors und der Forstverwaltung haben sich die Auffassungen in bezug auf den Naturschutz im Wald enorm entwickelt. Durch die Novellierung des Waldgesetzes von 1999 werden die ökologischen Bestimmungen in hohem Maße berücksichtigt.

\subsection{Die ökologische Bedeutung des Waldgesetzes}

Eine der Hauptzielsetzungen des flämischen Waldgesetzes von 1990 (als Ersatz für das belgische Forstgesetzbuch von 1854) war die Erhöhung des Naturschutzwertes im Wald. Die Maßnahmen waren jedoch hauptsächlich auf die öffentlichen Wälder ausgerichtet. Für den Privatwald waren immerhin höhere Fördermittel für den Anbau von einheimischen Laubbaumarten als für den Anbau von (nicht heimischen) Nadelhölzern vorgesehen.

Im öffentlichen Wald wurden die Zielsetzungen einer naturnahen Waldwirtschaft immer mehr betont. Es gab deutliche Bemühungen um die Erhöhung der Vielfalt der Wälder in der Praxis. 
Die Forsteinrichtungswerke müssen diese Ziele berücksichtigen. Das integrierte Schutzkonzept für den Waldbereich (Waldnaturschutzkonzept) umfasst den flächendeckend naturnahen Waldbau und, wie auch Kleinschmit (1999) betont, eine Verdoppelung des Laubbaumanteiles sowie die repräsentative Sicherung von natürlichen und kulturbedingten Lebensgemeinschaften in Waldgebieten und Sonderbiotopen. Eine große Anzahl von Wäldern wurden als Waldschutzgebiete ausgewiesen. Der Hohe Rat für Naturschutz muss eine Stellungnahme zu den die Belange der Natur berührenden forstlichen Maßnahmen abgeben und die Naturschutzverwaltung muss eine Stellungnahme zu geplanten Erstaufforstungen in Naturschutzgebieten verfassen.

Laut Waldgesetz von 1990 müssen die öffentlichen Wälder auf eine multifunktionelle Weise bewirtschaftet werden, mit einem Gleichgewicht zwischen den ökonomischen, ökologischen und sozialen Funktionen und unter Berücksichtigung der lokalen Begebenheiten.

Diese Entwicklung in Flandern ist mit jener in vielen anderen Ländern identisch, u.a. auch mit den Bestimmungen in Schweden, wo die novellierte Forstgesetzgebung festlegt, dass die Erhaltung der biologischen Vielfalt in den Wäldern ebenso wichtig ist wie die Holzproduktion (Richter, 1999). Die neue Forstgesetzgebung in Finnland ist auch Teil eines bereits lange bestehenden komplexen Prozesses, im Laufe dessen die finnische, herkömmlich auf kontinuierlicher Holzproduktion beruhende Forstwirtschaft in Richtung biologischer Vielfalt, ökologischer Nachhaltigkeit und Umweltschutz gelenkt wurde (Anon., 1997a).

\subsection{Schwerpunkt der Probleme}

Die Probleme zwischen der Forstwirtschaft und dem Naturschutz in Flandern entsprechen durchaus jenen, die von Glück (1998), Essmann (1989, 1993), Mann \& Essmann (1998) und Mann (1999) formuliert wurden.

- Waldbewirtschaftung (Gesamtkomplex, umfasst eine Fülle von Fragen, v.a. Berücksichtigung ökologischer und sozialer Aspekte);

- Arten- und Biotopschutz, Schutzgebietsflächen;

- Erstaufforstung;

- Definitionen von Verhaltensnormen, z.B. "Naturnähe", "Nachhaltigkeit", "ordnungsgemäße Forstwirtschaft";

- Flächenkompetenz der zuständigen Behörden;

- Forderungen nach vermehrter Ausweisung unbewirtschafteter Flächen.

Das flämische Naturschutzgesetz von 1997 wirft vor allem zwei Problemaspekte auf:

1. Die große Anzahl von horizontalen Maßnahmen, die auf das gesamte Land, also auch auf die Wälder, anwendbar sind, deren Anwendung jedoch nicht eindeutig geregelt ist. Dies betrifft u.a. die Bestimmungen, die es der flämischen Regierung ermöglichen, auf der gesamten Landesfläche Maßnahmen durchzuführen, die geeignet sind, die Umweltqualität zu wahren und den Naturschutz zu gewährleisten. Genau genommen bedeutet dies, dass die flämische Regierung praktisch jede Maßnahme treffen kann. Dem steht wiederum das Prinzip des "Nutzungsrechts" durch Land- und Forstwirtschaft entgegen. Gleichzeitig muss jedermann der Sorgepflicht Genüge lelsten und das Integrationsprinzip der europäischen Umweltpolitik 
anwenden. Hier stellt sich selbstverständlich wiederum die Frage: Was bedeutet dies konkret? In welchem Maß widersprechen sich besagte Bestimmungen einander?

2. Begriffe, die sich auf das qualitative Niveau der Waldbewirtschaftung beziehen, sind nicht abschließend definiert (siehe auch Mann, 1990).

Es ist derzeit nicht bekannt, welche Wälder in die Kategorie der VEN- und IVON-Gebiete fallen. Und es ist gleichfalls nicht bekannt, was die Folge einer "vorrangigen Naturschutzfunktion" sein wird. Die ökonomischen Folgen könnten jedoch erheblich sein. Diese Gebiete müssen zudem ausgewiesen werden, bevor die für diese Gebiete gültigen Behandlungsrichtlinien bekannt sind.

\section{Die Zuständigkeit der Forstverwaltung}

Das Waldgesetz von 1990 bestimmt ausdrücklich, dass die Forstverwaltung für die Umsetzung der Forstgesetzgebung in allen Wäldern zuständig ist, es sei denn dass die flämische Regierung dies anders entscheidet. Dieser Artikel wird durch den Naturschutz angefochten, weil die Naturschutzverwaltung die ausschließliche Zuständigkeit für Wälder mit dem Status eines Naturschutzgebietes für sich in Anspruch nehmen wollte. Hieraus ergaben sich Konflikte vor allem hinsichtlich der Bewirtschaftung von als Naturschutzgebiet ausgewiesenen Wäldern. Das Naturschutzgesetz von 1997 löste dieses Problem zugunsten des Naturschutzes: Die Forstverwaltung muss nur noch im Rahmen einer Stellungnahme zum Bewirtschaftungsplan dieser Wälder beteiligt werden.

Auf Druck der Naturschutzverwaltung wurde das Waldgesetz im Jahre 1999 durch weitere Bestimmungen ergänzt:

- Die nicht staatlichen Waldschutzgebiete werden weiterhin von dem Eigentümer bewirtschaftet. Vorher wurden alle Waldschutzgebiete von Amts wegen durch die Forstverwaltung bewirtschaftet.

- Im Gegensatz zu der ursprünglichen Gesetzgebung können Naturschutzverbände jetzt auch öffentliche Wälder betreuen. Diese Wälder müssen allerdings den Status eines Schutzgebietes haben.

\subsection{Die ökologische Waldfunktion}

Die ökologische Funktion des Waldes im engen Sinne, namentlich der Schutz der Flora und Fauna, wurde 1990 auf forstliche Weise erfült. Diese Bestimmungen waren nur für den öffentlichen Wald gültig. Übrigens war die Bedeutung des Leitbildes "ökologische Waldfunktion“ bzw. der Begriff "anzustrebende Zielsetzung" nicht klar.

Im Jahre 1999 wurde die ökologische Funktion im Waldgesetz komplett neu formuliert. Die forstwirtschaftliche Terminologie wurde durch eine typische Terminologie des Naturschutzes ersetzt. Im Bewirtschaftungsplan (Forsteinrichtung) aller Wälder, also auch bei Privatwald, muss angegeben werden, im welchem Maße die ökologische Funktion berücksichtigt wird. 


\subsection{Das Flämische Ökologische Netzwerk}

Der Aufbau des Flämischen Ökologischen Netzwerkes ist ein Hauptziel des Naturschutzgesetzes von 1997. In diesen Gebieten ist der Schutz der Natur die Hauptfunktion.

Es ist zu erwarten dass, im Gegensatz zu den ursprünglichen Absichten, zahlreiche Wälder in diese Schutzkategorie fallen. Es ist selbstverständlich auch zu erwarten, dass die ökonomische Funktion in diesen Gebieten eingeschränkt wird. Aber, wie bereits gesagt, die Ausweisung dieser Gebiete muss noch erfolgen und die entsprechenden Rechtsverordnungen müssen noch erlassen werden. Auch die Frage der Entschädigung ist noch nicht geklärt. Deshalb sind noch immense Konflikte zwischen Waldeigentümern und Naturschutz zu erwarten.

Diese Konflikte werden wahrscheinlich durch die europäische Vogelrichtlinie und die Benennung der Gebiete nach FFH-Richtlinie noch verstärkt werden. Letztere wurden vor einigen Jahren ohne besondere Bewirtschaftungseinschränkungen ausgewiesen. In naher Zukunft müssen in diesen Gebieten jedoch ebenfalls konkrete Maßnahmen durchgeführt werden.

\subsection{Weitere Instrumente}

Das Naturschutzgesetz 1997 erwähnt zugleich eine Reihe von Instrumenten, die neu für den Waldeigentümer sind:

- Die Genehmigung: sie ist notwendig für die Änderung der Vegetation. Sie ist jedoch nicht erforderlich wenn ein bewilligter Bewirtschaftungsplan besteht und wenn der Eingriff lediglich eine normale Pflegemaßnahme darstellt. Es ist jedoch nicht eindeutig ob z.b. eine Endnutzung als "normale Pflegemaßnahme" anzusehen ist.

- Vertragsnaturschutz. Dieses Instrument wurde bisher in der Forstwirtschaft Flanderns noch nicht angewandt. Schwerpunkt sind hierbei eigentlich auch eher Maßnahmen im landwirtschaftlichen Bereich. In der Forstwirtschaft erscheinen direkt finanziell unterstützende Maßnahmen attraktiver.

Als typisches Beispiel für den Vertragsnaturschutz wird auf die Bekämpfung von Prunus serotina verwiesen, eine eher spezielle und kurzfristige Maßnahme.

- Fördermittel: die flämische Regierung kann Fördermittel für Maßnahmen der Förderung einer natürlichen Entwicklung vergeben. Darüber hinaus kann sie ebenfalls Entschädigungen für Einkommensverluste gewähren. Ein typisches aktuelles Beispiel sind Ausgleichzahlungen bei der Ausweisung von Schutzgebieten. Diese können in einigen Fällen sehr hoch sein. Sie sind fast immer höher als die sonstigen Gewinne, die ein Waldeigentümer erzielen kann, weil in der Regel rote Zahlen geschrieben werden. Wagner und Gundeman (1996) diskutieren die Ausgleichszahlungen an Waldeigentümer für Naturschutz und Hochwasserschutz in Deutschland.

In Finnland sind ähnliche unterstützende Maßnahmen in der Forstgesetzgebung selbst vorgesehen (Anon., 1997a). Eine Subvention kann hier auch für eine besondere 
Naturschutzpflegemaßnahme im Wald gewährt werden, entweder als Umweltförderung oder als Naturschutzprojekt.

Betreffs der Finanzierung des Waldnaturschutzes kommt Krott (1997) zu der Schlussfolgerung, dass der Ressourceneinsatz des Waldeigentümers für den Waldnaturschutz vom angestrebten Naturschutzstandard, der Effizienz der Leistungserstellung sowie der Verteilung der Kosten zwischen Waldeigentümer und sonstigen Beteiligten abhängt.

- Grunderwerb: die flämische Regierung sowie anerkannte, landespflegerisch tätige Naturschutzverbände haben unter bestimmten Umständen ein Vorkaufsrecht. Dieses Vorkaufsrecht war bei der Erörterung des Waldgesetzes im Jahre 1990 abgelehnt und auch bei der Novellierung 1997 nicht mehr eingeführt worden.

\subsection{Besondere Bestimmungen}

- Umwandlung

Das Naturschutzgesetz von 1997 verbietet prinzipiell Umwandlungen. Sie sind nur noch zulässig für Zwecke des öffentlichen Gemeinwohls. Dies wurde damals im waldarmen Flandern als eine sehr positive Errungenschaft angesehen.

Diese Bestimmung wurde jedoch durch ein spezielles Gesetz vom Jahre 1999 wieder geändert, sodass die Bestimmungen im novellierten Waldgesetz in der Fassung von 1999 aufgeweicht wurden. Das allgemeine Prinzip des Umwandlungsverbots wird erhalten, aber eine Reihe von Ausnahmen sind ermöglicht worden. Übrigens ist das vorgeschlagene System von Ausgleichs- und Ersatzmaßnahmen wahrscheinlich nicht effizient, weil diese lediglich im freien Raum vorgenommen werden können. Wahrscheinlich werden die erforderlichen Grundstücksflächen in der Regel nicht zur Verfügung stehen.

Sehr bemerkenswert ist jedoch, dass das Naturschutzgesetz die Bestimmungen zur Umwandlung in Naturschutzgebieten sehr großzügig handhabt. Naturschützer betrachten in der Tat die Wälder in vielen Fallen als Hindernis für die Entwicklung von für den Naturschutz wertvollen Flächen. Die Frage stellt sich jedoch in welchem Maße diese Praxis in sehr waldarmen Gebieten wie Flandern durchgesetzt werden kann, ohne dem Prinzip der Walderhaltung zu widersprechen.

- Exotenbewirtschaftung

Obwohl dem Naturschutz an dem Erhalt und der Förderung autochthoner Vorkommen sehr gelegen ist, wird diesem Problem im Naturschutzgesetz kaum Aufmerksamkeit gewidmet. Im geänderten Waldgesetz von 1999 wird zwar erwähnt, dass die autochthonen Baumarten gefördert werden müssen, der Begriff "standortsgemäße Baumart", eingeführt im Waldgesetz von 1990, wird jedoch weggelassen. Es bleibt jedoch völlig undurchsichtig in welchem Maß z.B. der Anbau von Exoten verboten werden kann. Kann dies auf Basis der verschiedenen Bestimmungen des Naturschutzgesetzes zur Förderung einer natürlichen Entwicklung geschehen? 
- Wasserhaushalt

Wie bekannt, legt der Naturschutz großen Wert auf die Wiederherstellung des natürlichen Wasserhaushaltes. Dieses Leitbild wird häufig erwähnt. Vertragsnaturschutz ist möglich um in Waldgebieten den Grundwasserspiegel wieder auf ein natürlicheres Niveau anzuheben.

Kapuscinski (1997) stellt allgemein fest, dass man zu den für den Naturschutz besonders wertvollen Wäldern die schwer zugänglichen Wälder wie z.b. Sumpfwälder (oder Steilhänge und Gebirgskämme) zählt, welche in der Vergangenheit von einer Nutzung verschont blieben. Sie stellen heute oft die einzigen Überbleibsel der natürlichen Waldgemeinschaften dar, in welchen noch viele seltene oder vom Aussterben bedrohte Pilz-, Pflanzen- und Tierarten vorkommen.

- Artenschutz

Naturschutz kann auch der Forstwirtschaft Verpflichtungen zum Schutz gefährdeter Arten auflegen (Hickman, 1996). Dies ist z.b. möglich im Zusammenhang mit Maßnahmen zur Verbesserung des Wasserhaushalts oder der Förderung der biologischen Vielfalt in den Wäldern.

\subsection{Bewirtschaftungsplan}

Das Waldgesetz von 1990 hat auch den Privatwaldeigentümern die Verpflichtung auferlegt einen Bewirtschaftungsplan zu erstellen. Diese Bestimmung erweist sich heute als ein besonders geeignetes Mittel um den durch das Naturschutzgesetz auferlegten Verpflichtungen zu entrinnen. In der Tat ist bisher fast jede Maßnahme zulässig, die in einem genehmigten Bewirtschaftungsplan enthalten ist.

Die Frage stellt sich jedoch welchen Verpflichtungen der Bewirtschaftungsplan in Zukunft nachkommen muss, wenn er die Bestimmungen des VEN und der Behandlungsrichtlinien berücksichtigen muss. Übrigens wird die Naturschutzverwaltung ein Gutachten über Forsteinrichtungswerke in typischen Naturschutzgebieten verfassen müssen. Welche Einstellung wird sie dabei vertreten?

Die allgemeinen Schlussfolgerungen zum Verhältnis zwischen Naturschutzgesetz und Waldgesetz liegen auf der Hand:

1. Es gibt eine Reihe von Überlappungen zwischen dem Waldgesetz von 1990, revidiert im Jahre 1999, und dem Naturschutzgesetz von 1997.

2. Das Waldgesetz ist geprägt von einer bemerkenswerten Rangstellung der Naturschutzfunktionen im Wald.

3. Der Einfluss des Naturschutzes auf die Forstpolitik, die Forstgesetzgebung und die Waldbewirtschaftung ist von großer Bedeutung.

4. Das Naturschutzgesetz kann zukünftig einen noch viel größeren Einfluss auf die Waldbewirtschaftung ausüben.

5. Es gibt im Naturschutzgesetz viele Quellen für Konflikte zwischen dem Waldsektor und dem Naturschutzsektor. Sie können zu einer scharfen Auseinandersetzung zwischen beiden Bereichen führen, in der vor allem die Privatwaldbesitzer dle Betroffenen sein werden. 
6. Konflikte zwischen den Sektoren werden unvermeidlich zu einer Demotivation der Waldeigentümer und in der Folge zu einer Wertminderung des Waldeigentums führen.

Um eine zweckmäßige Forstpolitik für das 21. Jahrhundert zu entwickeln wird es absolut notwendig sein eine effiziente Zusammenarbeit zwischen dem Wald- und dem Naturschutzsektor aufzubauen. Auch Zimmermann (1991) kam zu diesem allgemeinen Schluss für die Schweiz, wo ebenfalls einerseits eine Forstgesetzgebung besteht die sich hauptsächlich mit Forstschutz und Forstökonomie beschäftigt, andererseits Ökologie, Erholung und Naturschutz durch andere Gesetzgebungen geregelt werden.

Forstleute besaßen lange Zeit die alleinige Verantwortung für die qualitative und quantitative Walderhaltung (Weber et al. 1998). Sie waren es gewohnt, die Abwägung der Nachhaltigkeit innerhalb des magischen Dreieckes zwischen "Ökonomie", "Ökologie" und "Soziales" weitestgehend alleine zu treffen, aufgrund eigener Erkentnisse und meist auch in bester Absicht (Anom., 1997b).

Seit dem Erdgipfel von Rio 1992 hat sich jedoch der Begriff "Partizipation" einen festen Platz in der politischen Diskussion über die Weiterentwicklung der Umweltpolitik erobert (Weber et al., 1998). Seitdem nehmen sich verschiedene Organisationen verstärkt forstlicher Themen an und werden damit zu neuen Akteuren der Forstpolitik. Das Interesse der Bevölkerung am Natur und Umweltschutz ist stark gewachsen. Dem wird im Auftrag des Staates durch die Forstverwaltungen auf einem gesetzlich normierten Mindestniveau entsprochen (Mann, 1999). Weitergehende Forderungen der Naturschutzverbände betreffen sowohl die technischen Methoden der Waldbewirtschaftung als auch formal die Verteilung forstpolitischer Zielsetzungs-, Planungs- und Kontrollkompetenzen. Diese Forderungen werden bislang hauptsächlich an die Forstverwaltung gerichtet. Die wachsende Zahl von Menschen, die den Zielen des Natur- und Umweltschutzes und den Forderungen der neuen sozialen Bewegungen nach mehr Partizipation positiv gegenüberstehen, stärkt den politischen Einfluss der Naturschutzverbände (vgl. Weber und Mann, 1997).

Mit Blick auf die hohen Anforderungen des Naturschutzes ist es doch bemerkenswert dass Mann und Essmann (1998) feststellen, dass die Forderungen des Naturschutzes nach vermehrter Ausweisung unbewirtschafteter Flächen und die erhöhten Anforderungen an die Art der Waldbewirtschaftung durch Biotop- und Artenschutz keine bedeutende Rolle in der Krise der Forstwirtschaft einnehmen.

Für eine gute Zusammenarbeit zwischen Forstwirtschaft und Naturschutz wäre jedoch eine wichtige Voraussetzung, dass der Naturschutz eine positive Einstellung gegenüber der Forstwirtschaft beweist und dass diese wiederum Schwächen zugeben kann. Jedenfalls ist das Naturschutzgesetz in Flandern ein sehr schwierig zu handhabendes Instrument.

Darüber hinaus ist, wie auch Oettingen-Spielberg et al. (1998) feststellen, die theoretische Basis stellenweise brüchig und das Datenmaterial sehr lückenhaft. 
Schließlich sollte in Flandern nicht nur der Naturschutz ein Partner der Forstwirtschaft sein, sondern auch die Landwirtschaft (Lust, 1996).

\section{Literatur}

Anon. (1997a). Neue Forstgesetzgebung für Finnland. AFZ/Der Wald, 4, 184.

Anon. (1997b). Forstwirtschaft im Spannungsfeld zwischen Naturschutz und Ökonomie. AFZ/Der Wald, 17, 909-912.

Essmann, H. (1989). Forstwirtschaft und Naturschutz. Forst und Holz, 14, 355-359.

Essmann, H. (1993). Unterschiede und Übereinstimmungen in Theorie und Praxis von Forstwirtschaft und Naturschutz. Allgemeine Forstzeitschrift, 11, 522-525.

Hickmann, C.A. (1996). Federal protection of threatened and endangered species: implications for forest management in the United States. Forstwissenschaftliche Beitrage, ETH-Zürich, No.16, 142-158.

Kapuscinski, R. (1997). Naturschutz in den Wäldern Polens. AFZ/Der Wald, 8, 422-423.

Kleinschmit, H. (1999). Naturschutzkonzept in den niedersächsischen Landesforsten. Forst und Holz, 54, Nr.20, 627-630.

Krott, M. (1997). Finanzierung des Waldnaturschutzes. Allg. Forst-u. J.-Ztg.,168. Jg., 11-12, 203-207.

Le Master, D.C., O'Leary, J.T. \& Sample, V.A. (1996). Forest service respons to changing public values, policies and legislation during the twentieth century in the United States. Forstwissenschaftliche Beitrage, ETH-Zürich, No. 16, 164-197.

Lust, N. (1996). Origin and development of the Flemish Forest Decree. Forstwissenschaftliche Beitrage, ETH-Zürich, No. 16, 215-226.

Mann, S. (1999). Konflikte in der deutschen Forstwirtschaft - Forstpolitische Schlussfolgerungen aus einer konflikttheoretische Analyse. Allg. Forst- u. J.-Ztg., 170. Jg., 2, 22- 28.

Mann, S. \& Essmann, H. (1998). Konflikte in der Forstwirtschaft in krisenhafter Zeit. Allg. Forstu. J.-Ztg., 169. Jg., 1, 4-14.

Richter, J. (1999). Silviculture and nature conservation in boreal Sweden. Forst und Holz, 54:3, 75-77.

Strijckers, P. (1999). De relatie tussen het Natuurbehoudsdecreet en het bos. Groene Band, $108,17-40$.

Wagner, S. \& Gundemann, E. (1996). Compensation payments to forest owners for nature and water protection measures in the Federal Republic of Germany. Forstwissenschaftliche Beitrage, ETH-Zürich, No.16, 304-328.

Weber, N. \& Schnappup, Ch. (1998). Partizipation-ein neues Grundprinzip in der Forstpolitik? Allg. Forst- u. J.-Ztg., 169. Jg., 9, 168-174

Zimmermann, W. (1991). Swiss legislation and social problems concerning forests. Italia Forestalia e Montana, 46:3,177-192

zu Oettingen-Spielbereg, A., Oettingen \& von Schilcher, F. (1998). Naturschutz und Wald in Deutschland. AFZIDer Wald, 16, 839-842. 